\title{
Investigation of inflammatory and allergic responses to common mold species: Results from in vitro experiments, from a mouse model of asthma, and from a group of asthmatic patients
}

\author{
M. Vincent ${ }^{1}$ (D) | P. Percier ${ }^{1}$ | S. De Prins ${ }^{1}$ | K. Huygen ${ }^{1}$ | G. Potemberg ${ }^{2}$ | \\ E. Muraille ${ }^{2,3}$ | M. Romano ${ }^{1}$ O. Michel ${ }^{4}$ O. Denis ${ }^{1}$
}

${ }^{1}$ Scientific Service Immunology, WIV-ISP, Bruxelles, Belgium

${ }^{2}$ Unité de Recherche en Biologie des Microorganismes, Laboratoire d'Immunologie et de Microbiologie, NARILIS, Université de Namur, Namur, Belgium

${ }^{3}$ Laboratoire de Parasitologie, Faculté de Médecine, Université Libre de Bruxelles, Bruxelles, Belgium

${ }^{4} \mathrm{Clinic}$ of Immunology and Allergology, $\mathrm{CHU}$ Brugmann, Université Libre de Bruxelles,

Bruxelles, Belgium

Correspondence

Olivier Denis, Scientific Service Immunology, WIV-ISP, Bruxelles, Belgium.

Email: odenis@wiv-isp.be

Funding information

Belgian Science Policy (BELSPO), Grant/Award

Number: IAP P7/32

\begin{abstract}
Most studies on molds focus on Alternaria alternata and Aspergillus fumigatus. Here, we report on inflammatory and allergenic properties of more typical indoor species Aspergillus versicolor, P. chrysogenum, C. cladosporioïdes, and C. sphaerospermum that were compared to A. alternata and A. fumigatus. In a mouse model, after intranasal instillation, A. alternaria, A. versicolor, and C. sphaerospermum induced the early recruitment of neutrophils and the strong expression of inflammatory markers in the bronchoalveolar lavages fluids. A. fumigatus also induced the early accumulation of neutrophils but with lower levels of inflammatory markers. Chronic treatment induced variable response according to species: P. chrysogenum and A. fumigatus appeared strong pro-allergenic inducers compared to A. alternata and C. sphaerospermum while A. versicolor and C. cladosporioides induced a mixed pro-allergenic/pro-inflammatory response. In mold-sensitized asthmatics, mold-specific Immunoglobulin E (IgE) were detected with an in-house dot-blot assay. A. fumigatus and A. alternata were the most frequent sensitizers. Altogether, P. chrysogenum, P. brevicompactum, C. sphaerospermum, and C. cladosporioides were the "major sensitizer" (defined as the strongest response against a single mold species) for almost $30 \%$ of the asthmatics. These results show that, not only A. alternata and A. fumigatus, but also indoor species have strong inflammatory and allergic properties and a harmful potency.
\end{abstract}

KEYWORDS

asthma, dot-blot assay, indoor molds, inflammatory properties of molds, mold sensitization,

mouse model of asthma

\section{1 | INTRODUCTION}

Molds are ubiquitous filamentous fungi able to colonize multiple environments. The majority of mold species are saprophytes, growing on most substrates, and recycling organic materials if enough moisture is available. So far, more than 112 different mold species have been recognized as important source of allergens that can cause respiratory diseases. $^{1}$

Fungal spores are metabolically inactive and can tolerate environmental stress. Depending on the species, the size of mold spores ranges from 2 to $50 \mu \mathrm{m}$ allowing their deposition into the airways. The external layer of the spores and the mycelium is a complex matrix structure 
with innate immune system stimulating properties such as the $\beta-1,3$ glucans recognized by $\mathrm{C}$-type lectin receptors (dectin-1 and 2, Mincle and mannose receptors) expressed on phagocytic cells, dendritic cells (DCs), neutrophils, and epithelial cells. The engagement of these Ctype lectin receptors triggers the activation of intracellular signaling pathways leading to the activation of inflammasomes and promotes inflammatory responses critically required to control fungal infections. Indeed, the activation of dectin-1 at the surface of DCs by A. fumigatus induces the respiratory burst and the production of inflammatory mediators, including tumor necrosis factor $\alpha$ (TNF- $\alpha$ ), various interleukins (IL-1 $\beta$, IL-6, IL-23), chemokines ligands (CCL-2 and CCL-3), and chemokine (C-X-C motif) ligand 1 (CXCL-1). ${ }^{2}$ In addition, dectin-1 knock-out mice have an increased mortality rate to an A. fumigatus challenge due to a poor neutrophil recruitment and an impaired fungal killing. ${ }^{3}$ These findings show that $\mathrm{C}$-type lectin receptors can activate innate immune cells, the first line of defense, and also modulate the development of the adaptive immunity through the differentiation of T-helper 1 (Th1) and T-helper 17 (Th17) cells, two critical cell subsets to mount a protective immune response against pathogenic fungi. ${ }^{4-6}$ These responses have been widely investigated with the pathogenic $C$. albicans and $A$. fumigatus but the relative contribution of $C$-type lectin receptors in the development of immune responses against nonpathogenic molds as for instance Penicillium spp, Cladosporium spp or A. alternata is lacking and the mechanisms involved are not formally identified yet.

The fungal cell wall is also composed of glycoproteins with allergenic properties. ${ }^{7}$ In addition, mold allergens are also found in enzymes released in the external environment during the germination process. $^{8,9}$ The lung epithelium and the dendritic cells present in the lung can recognize these allergens which initiates the allergenic process. Various signaling pathways, characterized by the secretion of IL-25, IL-33, and the activation of different innate immune cells, contribute to generate a pro-T-helper 2 environment in the lungs. This ultimately leads to the activation of nonprotective allergen-specific cells predominantly secreting characteristic Th2 cytokines such as IL-4, IL-5, and IL-13. ${ }^{10,11}$ These cytokines induce IgE production, cause eosinophilia, stimulate mast cells, promote leukocytosis, and provoke airway hyper reactivity. This immune activation in asthma patients leads to repeated attacks of breathlessness, cough, and wheeze occurring secondary to broncho-constriction in the setting of airway hyper-responsiveness, mucus hyper-secretion, and airway remodeling. ${ }^{12}$ However, in many patients with severe asthma, the lung inflammatory status is more heterogeneous and characterized by the presence of neutrophils and a production of IL-17. Therefore, it has been postulated that IL-17 may also play an active role in severe forms of the disease. ${ }^{13-15}$

Alternaria alternata and Cladosporium herbarum are the most common outdoor mold species. A large European study in asthmatics showed that the prevalence of sensitization to these two species increased significantly with increasing asthma severity. ${ }^{16}$ In addition, exposure to airborne spores of $A$. alternata represents a risk factor for respiratory failure underlining the association between asthma severity and sensitization to this species. ${ }^{17}$

Mold spores can likewise be present in the indoor environment, due to some housing activities or inhabitant behaviors, but

\section{Practical Implications}

- Both experimental results from a mouse model of asthma and data obtained from mold-sensitized asthmatics highlight the harmful properties of mold species commonly isolated indoor. The presence of these species should therefore be thoroughly investigated during home enquiries and future research work should focus on investigating their link with adverse respiratory health effects.

the association between their presence and adverse health effects is controversial. ${ }^{18,19}$ Choi et al. ${ }^{20}$ found no association between the presence of ergosterol, $\beta$-glucans, or culturable molds and indoor dampness with asthma and allergic diseases symptoms of children. Other work found a positive association between respiratory adverse effects including asthma development or exacerbation and residential dampness but not with quantitative mold assessments. ${ }^{21,22}$ Finally, other studies indicated that exposure to some mold species commonly contaminating damp dwellings were associated with increased exacerbations and asthma development in children. ${ }^{23,24}$ In these settings, A. versicolor, $P$. chrysogenum, $C$. cladosporioïdes, and C. sphaerospermum are among the most common airborne and dustborne species isolated indoors. $^{25-28}$

To document the hazardous nature of indoor mold species and define their allergenic potential, we analyzed the activating properties of C. cladosporioides, P. chrysogenum, C. sphaerospermum, and A. versicolor and compared them to those of the outdoor mold A. alternata and the classical allergenic mold A. fumigatus. The cytokine secretion profile was studied after bone marrow-derived dendritic cells (BMDCs) stimulation with the spores of the six species. We also analyzed both acute and chronic immune response in an experimental model of asthma established in mice chronically instilled intra-nasally with mold spores. In parallel, the prevalence of sensitization to 10 mold species frequently isolated in Western Europe ${ }^{25,28}$ was estimated in a population of moldsensitized asthmatics. Results underline the noxiousness of $A$. fumigatus and A. alternata but also of exposure to indoor mold species.

\section{2 | MATERIALS AND METHODS}

\section{1 | Mold strains, spores and extract preparations}

Alternaria alternata (strain 18586), C. cladosporioides (strain 9072 and 6032), P. chrysogenum (strain 1240), C. sphaerospermum (strain 22974), and A. versicolor (strain 18884) were obtained from the Belgian Co-ordinated Collections of Micro-organisms/Institut d'Hygiène et d'Epidémiologie-Mycologie (BCCM ${ }^{\mathrm{TM}} / \mathrm{IHEM}$ ) from the Wetenschappelijk Instituut Volksgezondheid-Institut Scientifique de Santé Publique (WIV-ISP, Brussels, Belgium). A. fumigatus (strain AF293) was obtained from the American Type Cell Culture (ATCC-Manassas, VA, USA). These molds were cultured at $27^{\circ} \mathrm{C}$ $\left(20^{\circ} \mathrm{C}\right.$ for $\mathrm{A}$. versicolor and A.fumigatus) on potato dextrose agar 
(BD Difco-Franklin Lakes, NJ, USA) plates, for 2 weeks before gently harvesting the spores with a cell scraper. Spores were diluted in phosphate-buffered saline (PBS) and counted with a hemocytometer.

Aqueous extracts from A. alternata, P. chrysogenum, A. fumigatus, and C. sphaerospermum were purchased from Greer laboratories $(\mu \mathrm{g} / \mathrm{mL}$; Greer Laboratories - Lenoir, NC, USA). A. versicolor and C. cladosporioides extracts were prepared from mycelial mats cultured during 3 weeks in liquid Czapek media. Mats were grinded in liquid nitrogen as described by Achatz et al. ${ }^{29}$ Protein concentration was measured according to the Bradford method, and extracts were stored at $-20^{\circ} \mathrm{C}$.

\section{2 | In vitro BMDCs stimulations}

Bone marrow-derived dendritic cells (BMDCs) were prepared as described. ${ }^{30}$ Briefly, C57BL/6 mice were sacrificed and the bone marrow was extracted from femurs by flushing with PBS. Cells were cultured $\left(5 \% \mathrm{CO}_{2}\right.$ at $\left.37^{\circ} \mathrm{C}\right)$ in $\mathrm{T} 75$ flask at a density of $4 \times 10^{6}$ cells per plate in RPMI-1640 medium supplemented with $10 \%$ fetal calf serum (FCS), $5 \times 10^{-5} \mathrm{~mol} / \mathrm{L} \beta$-mercaptoethanol, gentamycin, non-essential amino acids, sodium pyruvate, and glutamax (Thermo Fisher ScientificWaltham, MA, USA) and mouse recombinant granulocyte-macrophage colony-stimulating factor (GM-CSF) $10 \mathrm{ng} / \mathrm{mL}$ (ImmunotoolsFriesoythe, Germany). The culture medium was replenished after 3 days. BMDC's were collected after 7 days, seeded in 96-well plates $\left(2 \times 10^{5}\right.$ cells/well) and cultured for 24 hours with either lipopolysaccharide (LPS; $100 \mathrm{ng} / \mathrm{mL}$ ) or curdlan $(100 \mu \mathrm{g} / \mathrm{mL})$ or zymosan $(100 \mu \mathrm{g} /$ $\mathrm{mL})$ or various mold spores $\left(2 \times 10^{5}\right.$ spores, except for A. alternata $2 \times 10^{4}$ spores).

\subsection{Animals and immunizations}

C57BL/6 mice were obtained from the Elevage Janvier (Le Genest Saint Isle, France) or from the breeding facilities of the WIV-ISP and maintained under standard laboratory conditions. All experiments were performed in accordance with the local ethics committee and were approved under the file number 120724-01. Female mice aged 10-12 weeks at the beginning of experiments (five mice per group unless otherwise stated) were instilled intranasally (i.n.) with $10^{6}$ mold spores on three consecutive days (on Monday, Tuesday, and Wednesday) to study early responses. To study chronic responses, the same protocol was used during the first week followed by two instillations per week (on Mondays and Fridays) during 4 weeks and a final instillation the fifth week, on Monday. As instillation of $10^{6}$ A. alternata spores induced severe cachexia (related to strong TNF- $\alpha$ production), a lower concentration of $10^{5}$ spores was used. ${ }^{31}$ The control mice were instilled with PBS only. Mice were anesthetized with isofluran (Isoflo, Abbott Laboratories-North Chicago, IL, USA) before instillations. Mice were killed and sampling was performed 24 hours after the last instillation.

To induce a polyclonal IgE production, mice were immunized twice by intra-peritoneal (ip) injections of $2 \times 10^{6}$ spores diluted in PBS at 1-month interval and sera were collected 15 days after the second injection. ${ }^{32}$

\section{4 | Bronchoalveolar lavages}

Bronchoalveolar lavage (BAL) fluids were collected as described before. ${ }^{32}$ Total cell counts were determined with a hemocytometer, differential cell counts were obtained by examining at least 300 cells on cytospin slides stained with Diff-Quick (Dade Behring-Deerfield, IL, USA) and expressed as cells $/ \mathrm{mL}$.

\section{5 | Cytokine detection in supernatants and BAL fluids}

The concentration of TNF- $\alpha$, IL-1 $\alpha$, and IL-1 $\beta$, IL- 6, IL-12, and IL-23 produced in vitro by BMDC's was determined by ELISA (Ready-SET-Go ELISA-eBiosciences, San Diego, CA, USA). The concentration of IL-4, IL-5, IL-13, and IL-33 was measured in BAL fluids by ELISA (R\&D systems, Minneapolis, MN, USA). The concentration of TNF- $\alpha$, IL-6, and IL-17 was determined by Cytokine Beads Array (BD Pharmingen-San Diego, CA, USA). Samples were acquired on a FACScalibur and analyzed with the Flow Cytometric Analysis Program array software (BD Biosciences-Franklin Lakes, NJ, USA).

\section{6 | Total mouse IgE determinations}

Total serum IgE concentrations were determined using a sandwich ELISA as described. ${ }^{32}$

\section{7 | mRNA chemokine and cytokine quantification}

Total RNA was extracted from homogenized lung cells with Trireagent (Sigma-Aldrich-Saint Louis, MO, USA), 24 hours after the last instillation. cDNA was synthesized using the GoScript Reverse Transcription System (Promega-Madison, WI, USA). RT-qPCR was performed on the Stratagene Mx3000P (Agilent Technologies-Santa Clara, CA, USA) using a GoTaq qPCR Master Mix with BRYT Green (Promega). Each RT-qPCR amplification was performed in duplicate under the following conditions: $50^{\circ} \mathrm{C}$ for 2 minutes and $95^{\circ} \mathrm{C}$ for 10 minutes, followed by 40 cycles at $95^{\circ} \mathrm{C}$ for 15 seconds and $60^{\circ} \mathrm{C}$ for 1 minute. The list of forward and reverse primers used in this study is shown in Table S1. Hydroxymethylbilane synthase (HmBS) mRNA was used as reference housekeeping gene for normalization. The level of target mRNA, relative to the mean of the reference housekeeping gene, was calculated as previously described. ${ }^{33}$

\subsection{Evaluation of histological changes in the lungs}

For histology analysis, lungs were collected 24 hours after the last instillation and fixed by tracheal infusion of formalin. Tissues were kept in formalin for 2 days and then embedded in paraffin according to a protocol previously described. ${ }^{31}$ For microscope examination, tissues were sliced and then colored with hematoxylin-eosin-safran for the detection of lung inflammation and with periodic acid Schiff for the detection of mucus produced by goblet cells. The lung preparation steps for histology analysis were conducted in Unité de Recherche 
en Physiologie Moléculaire (URPhyM), NARILIS, Université de Namur (laboratory of Pr. Y. Poumay).

\section{9 | Asthmatic subjects}

Asthmatic patients were enrolled in the Immuno-allergology Unit of Centre Hospitalier Universitaire (CHU) Brugmann (Brussels). The group consisted of 32 asthmatics, with a positive skin prick test (SPT) and/or CAP (cellulose acetate membrane precipitin) test for A. fumigatus and/or A. alternata and/or P. chrysogenum and/or C. herbarum. Asthma was diagnosed according to the Global Initiative for Asthma (GINA) guidelines. ${ }^{34}$ SPTs were carried out using a standardized procedure with reagents from Stallergènes (Antony-France). Positive (histamine and codeine) and negative (allergen diluent) controls were used. Weal size was measured after 20 minutes, and the result was considered positive if the weal was at least $4 \mathrm{~mm}$ greater than the negative control. CAP tests were performed in the Immunology laboratory at $\mathrm{CHU}$ Brugmann with the Phadia 1000 FEIA analyzer (Thermo Fisher Scientific) against A. fumigatus, A. alternata, P. chrysogenum, and C. herbarum. According to the manufacturer, the test has a limit of detection of $0.1 \mathrm{kU} / \mathrm{L}$ and a positivity threshold of $0.35 \mathrm{kU} / \mathrm{L}$. A written informed consent was obtained for each patient, and the study protocol was approved by the local Ethics Committee (CE2011/71).

\subsection{0 | Human mold specific IgE detection}

A dot-blot assay was developed in our laboratory for the detection of specific IgE. ${ }^{35}$ Briefly, mold extracts, diluted in borate buffer $(\mathrm{pH}$ 10), were coated on a nitrocellulose membrane (Amersham Protran 0.45 NC; GE Lifesciences-Chicago, IL, USA; 5 and $50 \mu \mathrm{g} / \mathrm{mL}$ for commercial and in-house extracts, respectively) using a 96-well dot-blot system (Minifold ${ }^{\circledR}$ I Dot-Blot System; Whatman Saint Louis, MO, USA). The membrane was removed from the device and blocked in 1\% bovine serum albumin (BSA; Sigma-Aldrich) in tris-buffered saline (TBS) for 1 hour at room temperature before being incubated with patient's sera (dilution $1 / 5$ in TBS-Tween20 0.1\%-BSA 0.1\%. 1.5 hours. Room temperature). The membrane was then incubated for 1.5 hours at room temperature with rat monoclonal anti-human IgE coupled to horseradish peroxidase (HRP; LO-HE 17-HRP \& LO-HE 10-HRP_ Institute of Experimental and Clinical Research, Université Catholique de Louvain, Belgium) diluted 1/5000 in TBS-Tween20 0.1\%-BSA $0.1 \%$. Between each incubation step, excess antibodies were removed by three successive washes with TBS for 3 minutes and then three times with TBS-Tween20 0.05\% for 5 minutes and then again three times with TBS for 3 minutes. The membrane was developed by addition of Amersham ECL Prime reagent (GE Lifesciences), and readings were performed on a bioanalyzer CCD-Camera (LAS 4000Mini; GE Lifesciences) and analyzed with the Image Quant TL software (GE Lifesciences). Two positive controls were used. The Non-WHO Human anti-Aspergillus fumigatus IgE Reference Serum (Product number 78/545 National Institute for Biological Standards and Controls-NIBSC, Hertfordshire-UK; concentration 25.6 kU/L measured by ImmunoCAP) and the serum of a volunteer with documented sensitizations to several mold species. Two sera that had shown no reactivity against any mold of the panel were used as negative controls. The background was estimated based on the signal from an uncoated membrane (sample dilution buffer). The results, obtained as pixel counts, were then converted into indexes to standardize their expression with the following formula:

$$
\text { INDEX }=\frac{\text { (pixel count for the tested serum }- \text { background })}{\text { (pixel count for the first negative control }- \text { background })} .
$$

For each mold extract, positivity thresholds were established from 43 serial analyses of the second negative control serum. Positivity thresholds were expressed as the mean index response obtained for each extract +3 standard deviations. For each positive serum, the "major sensitization," defined as the response against a single extract showing the highest difference as compared to the variation of response of the negative control was calculated with the following formula:

$\Delta$ for each extract $=$

(experimental serum index - index of the second negative control serum) standard deviation of the second negative control serum

This formula was used because it not only considers the gap between the response against the serum and the negative control for a certain mold extract but also the variation of the response. The positivity threshold of the dot-blot was calculated based on a dilution curve of the Non-WHO Human Reference Serum anti-Aspergillus fumigatus on a A. fumigatus extract coated membrane and is of $0.418 \mathrm{kU} / \mathrm{L}$. As no reference serum exists for other mold species, we had to assume that the sensitivity was comparable for other species.

\subsection{1 | Statistical analysis}

Mann-Whitney test was used for comparisons between two groups (mold spores vs RPMI/PBS). Cell recruitment in the BAL fluids was compared between mold species with one-way ANOVA and KruskalWallis test. Correlation between neutrophils count and CXCL2 expression was calculated with the Spearman correlation test. Significance was assumed at a $P$ value below .05. Data were obtained from at least two separate experiments. Analysis was performed with GraphPad Prism version 6.01.

\section{3 | RESULTS}

\section{1 | Inflammatory responses induced by the mold spores}

The in vitro inflammatory properties of spores from indoor mold species (C. cladosporioides, P. chrysogenum, C. sphaerospermum, and A. versicolor) were compared with two frequently studied species, A. alternata and A. fumigatus. Murine BMDCs were cultured in the presence of spores from these six species, and the production of inflammatory cytokines and chemokines released in the culture supernatant was analyzed. As shown in Figure 1, all tested mold species except $A$. versicolor induced a substantial production of TNF- $\alpha$. 
FIGURE 1 In vitro inflammatory cytokine production by BMDCs in response to mold spores. $2 \times 10^{5} \mathrm{BMDCs}$ from C57BL/ 6 mice were incubated with RPMI medium alone, LPS (100 $\mathrm{ng} / \mathrm{mL}$ ), zymosan $(100 \mu \mathrm{g} / \mathrm{mL})$ or various mold spores $\left(2 \times 10^{5}\right.$ spores except $2 \times 10^{4}$ spores for A. alternata). The concentration of each cytokines was measured in culture supernatants after a $24-\mathrm{h}$ culture at $37^{\circ} \mathrm{C}$. Results represent cytokine concentrations from five mice tested in duplicate $(\dagger P<.05$, $\S P<.01, ¥ P<.001, \neq P<.0001)$
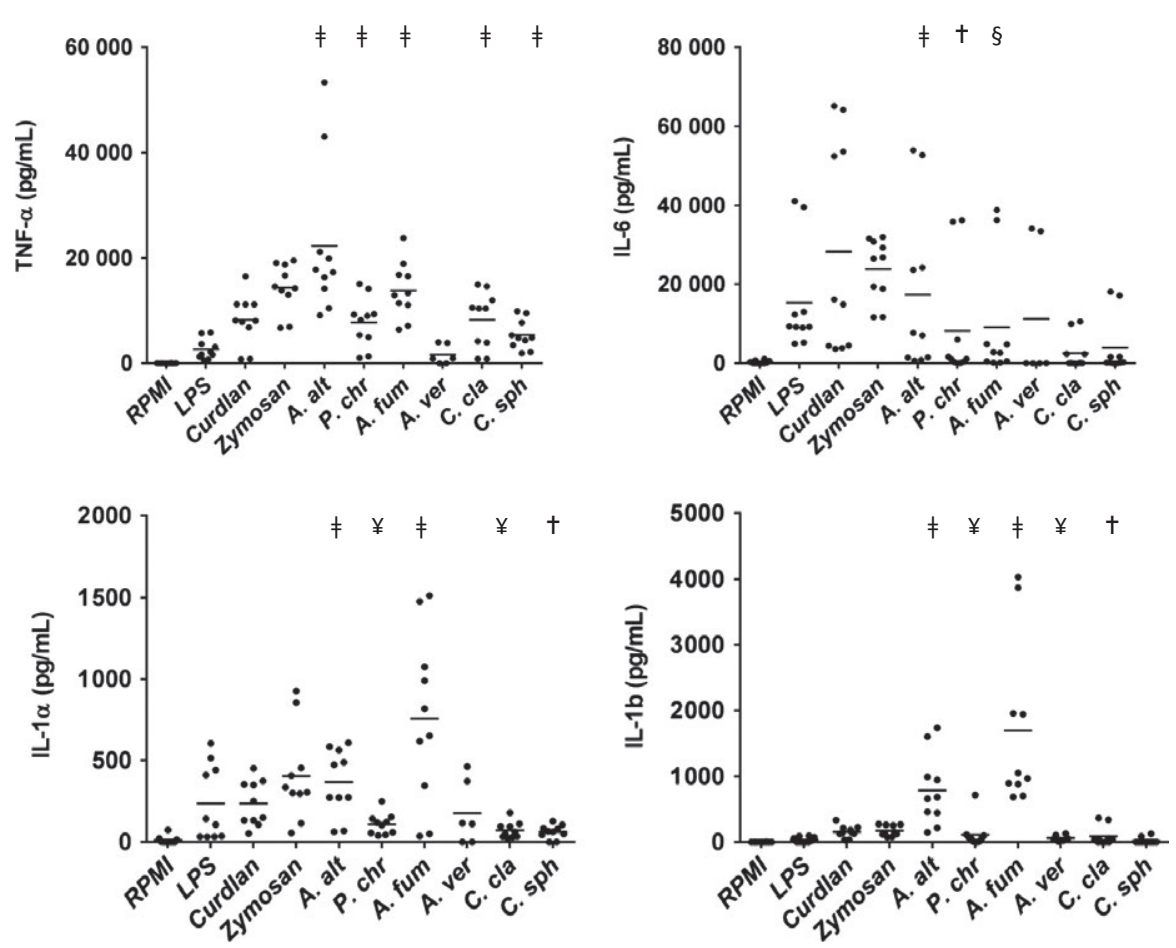

A. fumigatus and A. alternata induced also a very significant and strong production of IL- 6 , IL- $1 \alpha$, and IL- $1 \beta$ while other species elicited lower and variable production of these cytokines (Figure 1). None of the six tested species stimulated the production of IL-12. A. fumigatus and A. alternata triggered a significant albeit low production of IL-23 (data not shown).

The acute inflammatory properties of these species were also examined in vivo in C57BL/6 mice, 24 hours after three (once a day) intranasal instillations of the spores. In the BAL fluids, all mold spores induced a recruitment of macrophages. A. versicolor, C. sphaerospermum, A. alternata, and A. fumigatus elicited stronger neutrophil recruitment than $C$. cladosporïoides while $P$. chrysogenum only induced a minimal accumulation (Figure 2). The patterns of TNF- $\alpha$, CXCL-2, and IL-1 $\alpha$ mRNA lung expression were similar. These three cytokines were strongly induced by A. alternata, C. sphaerospermum, and A. versicolor. Other species such as A. fumigatus, C. cladosporioïdes, and P. chrysogenum elicited low but significant responses of these cytokines (Figure 3). The recruitment of neutrophils in BAL fluids was positively correlated with the lung expression of CXCL-2 $\left(r^{2}=.6597 ; P=.02\right.$; Figure 4$)$ indicating a match between cytokines and cells responses.

The expression of IL-33 in the lungs was also investigated. We found that the spores of the six mold species upregulated lung IL-33 with P. chrysogenum and C. sphaerospermum spores inducing a lower expression than spores of the four other species (Figure 3).
FIGURE 2 Early inflammatory cell recruitment in the bronchoalveolar lavage fluids of mold spore-treated mice. C57BL/6 mice were instilled daily during 3 days with PBS or various mold spores $\left(10^{6}\right.$ spores except $10^{5}$ spores for A. alternata), and the BAL fluids were collected $24 \mathrm{~h}$ after the last instillation. Results show the cell counts, per cell type for each mold species and were obtained from two independent experiments with five mice per group $(† P<.05, \S P<.01, ¥ P<.001, \neq P<.0001)$
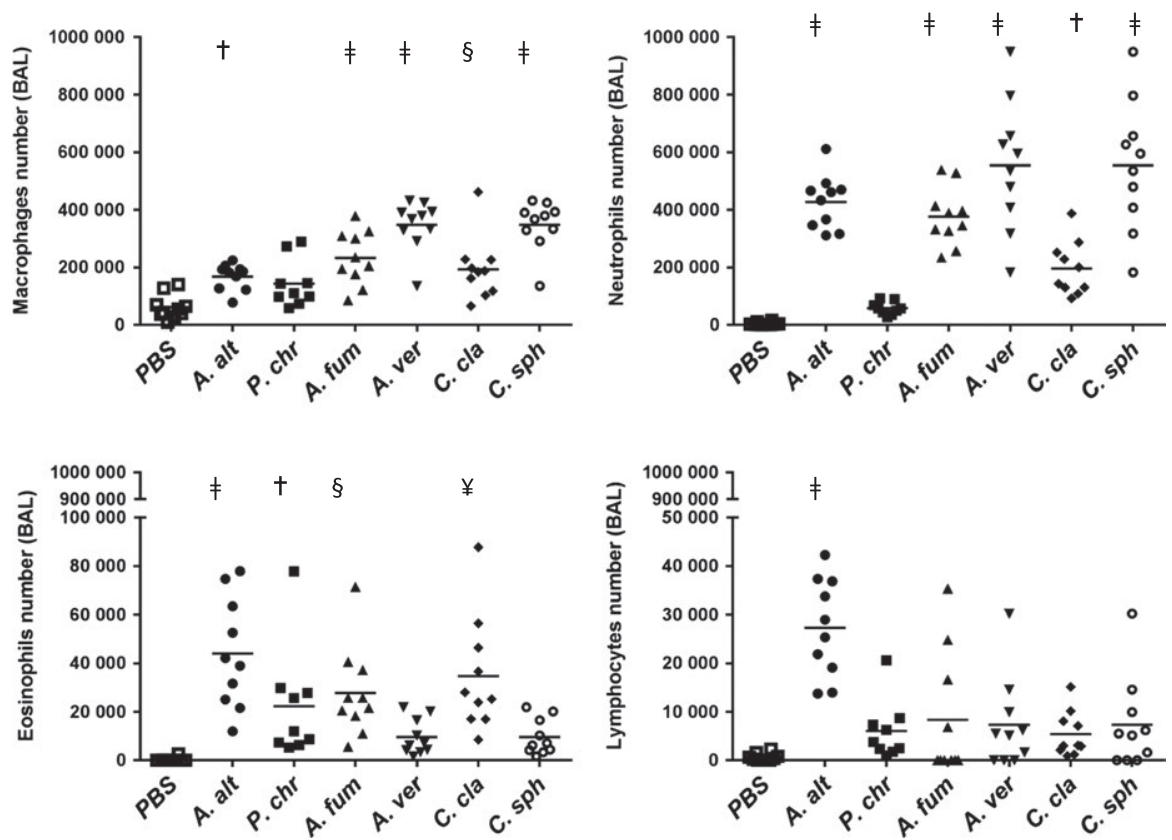

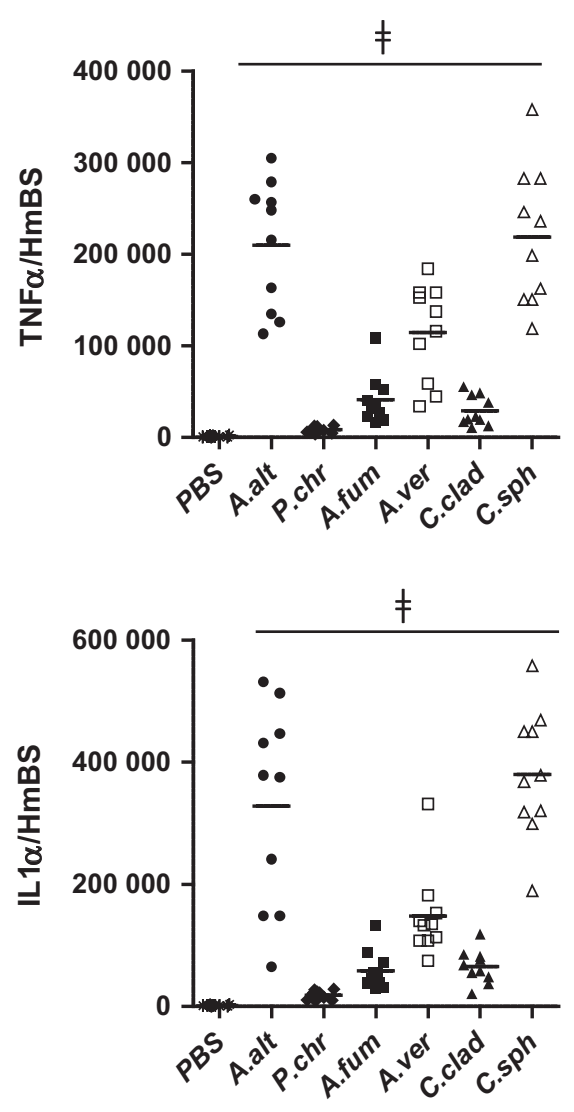
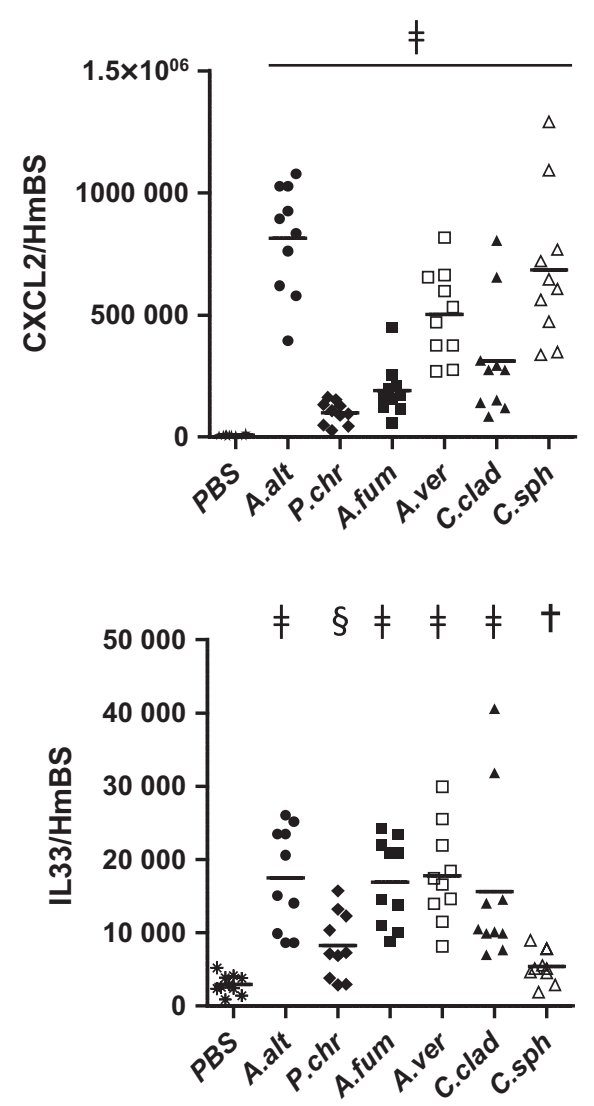

FIGURE 3 Analysis of the early cytokine/chemokine lung mRNA expression after stimulation with mold spores. C57BL/6 mice were instilled daily during 3 days with PBS or various mold spores $\left(10^{6}\right.$ spores except $10^{5}$ spores for A. alternata). Twenty-four hours after the last instillations, total RNA was extracted from the lungs, and transcriptional levels of the indicated cytokines were analyzed by quantitative RT-PCR. Results are pooled from two independent experiments with five mice per group. Significant differences between mold-treated mice vs control PBStreated mice $(\dagger P<.05, \S P<.01, ¥ P<.001$, $\neq P<.0001)$

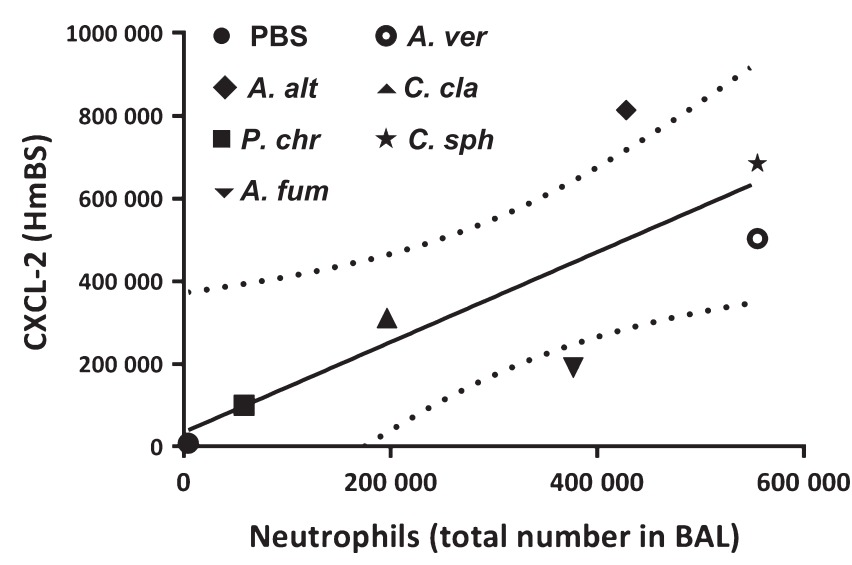

FIGURE 4 Analysis of the correlation between neutrophil recruitment and CXCL2 expression in BAL fluid. The Spearman correlation test was used to study the correlation between neutrophil recruitment in the BAL fluids and the level of lung CXCL2 expression after three daily instillations of the indicated mold spores. Values plotted on the graph represent average counts from two independent experiments with five mice per group. Neutrophil recruitment and CXCL2 expression after mold stimulation were positively correlated $\left(r^{2}=.6597 ; P=.02\right)$

\subsection{Allergic Th2 responses induced by the mold spores}

As shown in Figure 5, all species except $A$. versicolor induced significant IgE serum levels. A. fumigatus and C. cladosporïoides generated

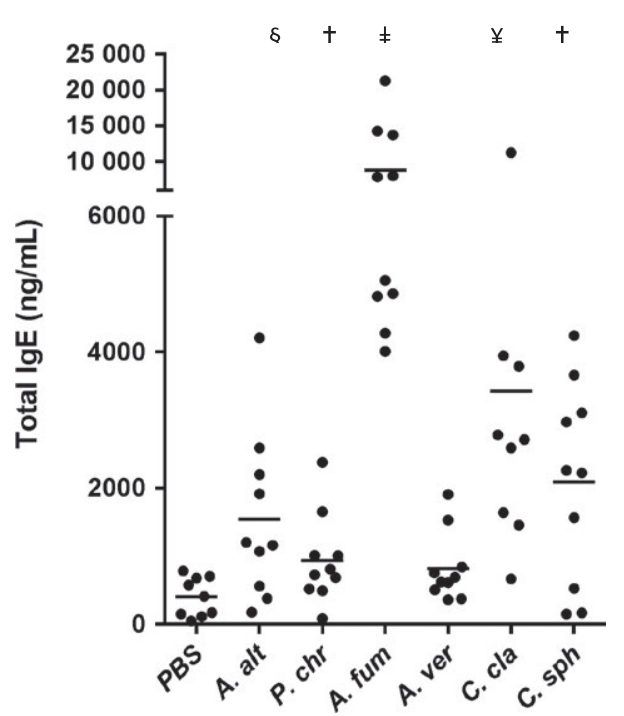

FIGURE 5 Detection of total serum IgE levels induced by mold spore immunization. C57BL/6 mice were immunized intraperitoneally twice at 1-month interval with $2 \times 10^{6}$ mold spores. Mice were bled 15 days after the boost. Data are from two independent experiments with five mice per group. Significant differences between IgE values of mold-treated mice vs control PBS-treated mice $(\dagger P<.05, \S P<.01$, $¥ P<.001, \neq P<.0001)$

the strongest responses. Cellular recruitments were analyzed in the BAL fluid 24 hours after the last instillation of a 5-week exposure scheme with the spores. Compared to the acute inflammatory response analyzed 24 hours after three intranasal instillations, a chronic 
treatment during 5 weeks led to an additional recruitment of eosinophils. A. fumigatus, P. chrysogenum, A. versicolor, and C. cladosporïoides induced a stronger recruitment of eosinophils than the two other species. A. alternata and C. sphaerospermum recruited more neutrophils than the other species and led to a balance between neutrophils and eosinophils. In addition to neutrophils and eosinophils, macrophages and lymphocytes were also recruited in the BAL fluids of mice treated with these six species (Figure 6).

In parallel, the expression of five Th2-related cytokines and chemokines, IL-4, IL-5, IL-13, CCL-11, and CCL-24 was strongly increased in the lungs of mice treated with all six mold species. A. alternata induced less CCL-11 than the other species while C. sphaerospermum had a tendency to produce less IL-4. The expression of IFN- $\gamma$ was also quantified (Figure 7) and the ratio IL-4/IFN- $\gamma$ calculated (Supporting Information). Results showed that $P$. chrysogenum was the species with the highest IL-4/IFN- $\gamma$ ratio while A. alternata had the lowest. The IL-4/IFN- $\gamma$ ratio of the four other species was intermediate.

Lung mRNA expression and BAL fluid concentrations of IL-17 and TNF- $\alpha$ are shown in Fig. S2. C. cladosporïoides and C. sphaerospermum were the main stimulators of TNF- $\alpha$ while $A$. versicolor, $C$. cladosporioides, and C. sphaerospermum were the main inducers of IL-17. A. alternata was also shown to increase the concentration of TNF- $\alpha$ in the BAL fluid. Noteworthy, A. fumigatus induced neither TNF- $\alpha$ nor IL-17 during this chronic phase. P. chrysogenum also failed to induce a production of TNF- $\alpha$.

A summary of all the findings obtained in the mouse model is presented in the Table S2.

To perform further analysis of the lung inflammation, an histopathological examination was conducted. Control PBS-treated mice showed no sign of inflammation. In contrast, mice instilled with the spores showed an important accumulation of inflammatory cells. P. chrysogenum, A. fumigatus, A. versicolor, and C. cladosporioïdes induced a recruitment mainly in the peri-vascular and peri-bronchial areas with numerous eosinophils. An accumulation of large macrophages in the lung parenchyma was also observed. After exposure to A. alternata and C. sphaerospermum, inflammation was more diffuse and fewer eosinophils were observed. Noticeably, in mice exposed to $P$. chrysogenum, the structure of bronchial epithelium was not very well-preserved and muscular hypertrophy was detected. Periodic acid Schiff (PAS) staining shows an intense production of mucus in mice exposed to mold spores. Of note, this production appeared less important after exposure to C. sphaerospermum (Figure 8).

\subsection{Analysis of the sensitization pattern to mold species in a group of asthmatics}

The presence of specific lgE against the 10 tested mold species was investigated in a group of asthmatic patients with positive SPT and/ or CAP test to commercial mold extracts. We used a dot-blot assay developed in our laboratory and worked with in-house mold extracts or commercial preparations that both differ from those usually used for SPT and CAP tests. Among these 32 patients, we found 25 patients with IgE reactivities against at least one mold of the panel. IgE against $A$. fumigatus and $A$. alternata were the most common, detectable in more than $40 \%$ of the asthmatics. In contrast, IgE against $C$. cladosporïoides, A. versicolor, and A. flavus were less common (less than $10 \%$ of the asthmatics). IgE antibodies against the other mold species were present in $20 \%-30 \%$ of the sera (Figure 9).

Cross-reactions are frequent in immune responses against mold antigens and have been reported in the literature. ${ }^{36,37}$ Our concern was therefore to attempt to identify, in the sera showing more than one positive response, the "major sensitization." It was defined as the antibody response with the highest difference as compared to the variation of response of the negative control. Using this definition, we
FIGURE 6 Late inflammatory cell recruitment in the bronchoalveolar lavage fluids of mold spore-treated mice. C57BL/6 mice were instilled daily during 3 days and then twice a week during 4 more weeks with PBS or various mold spores $\left(10^{6}\right.$ spores except $10^{5}$ spores for $A$. alternata). Mice were instilled again the next Monday and BAL fluids were collected $24 \mathrm{~h}$. Results show cell counts per cell type for each mold species. Data are from two independent experiments with five or six individual mice (A. versicolor, $C$. sphaerospermum, and C. cladosporioides group) per group $(\dagger P<.05$, $\S P<.01, ¥ P<.001, \neq P<.0001)$
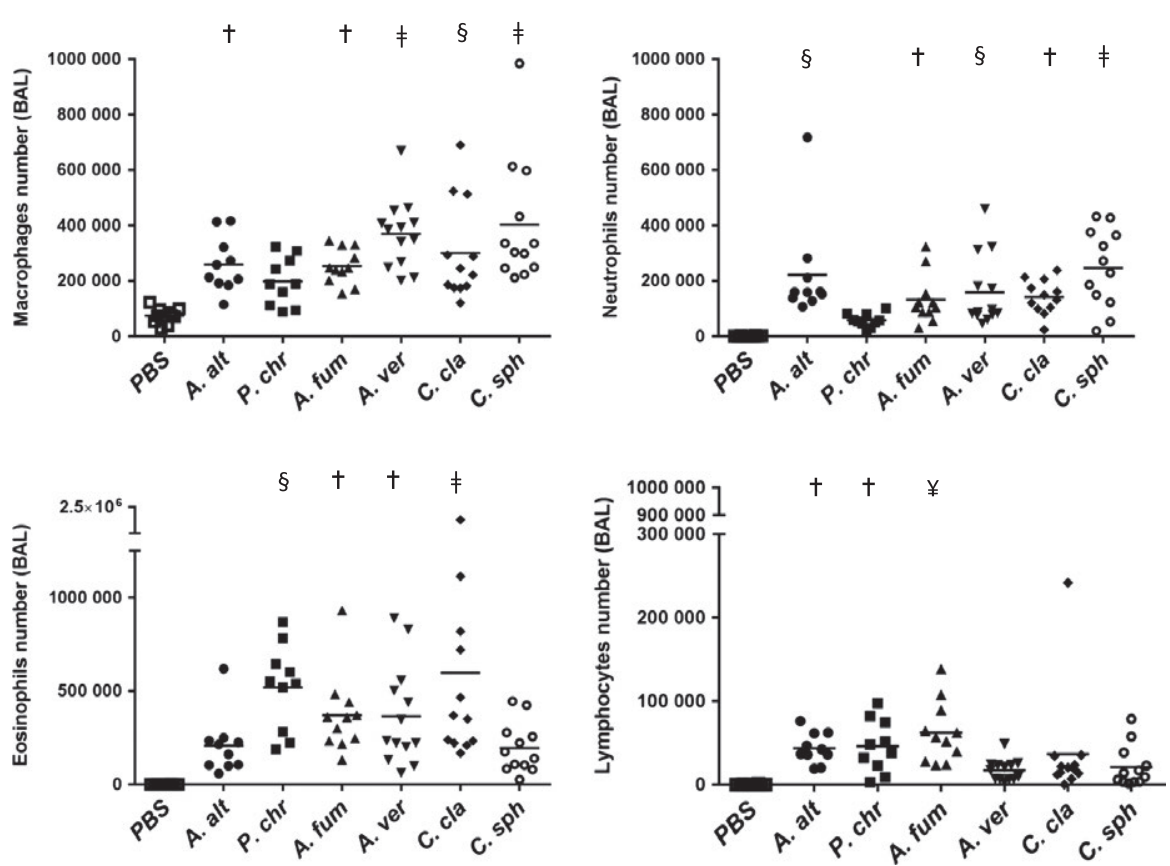
(A)
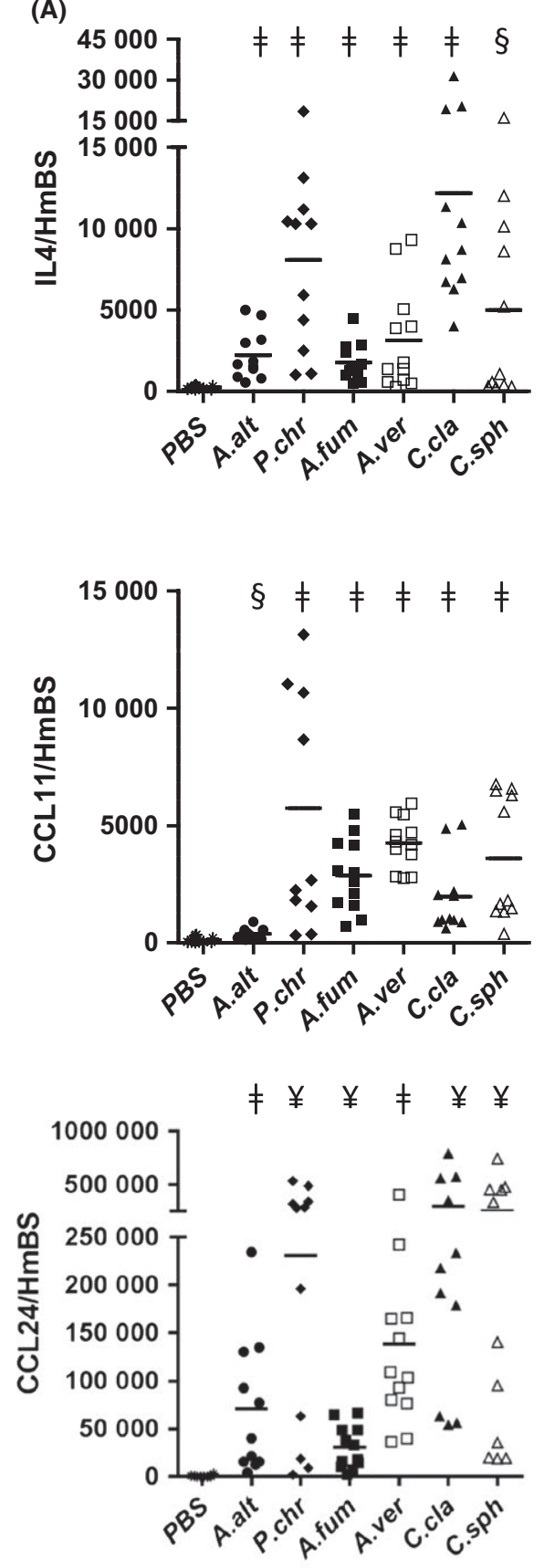
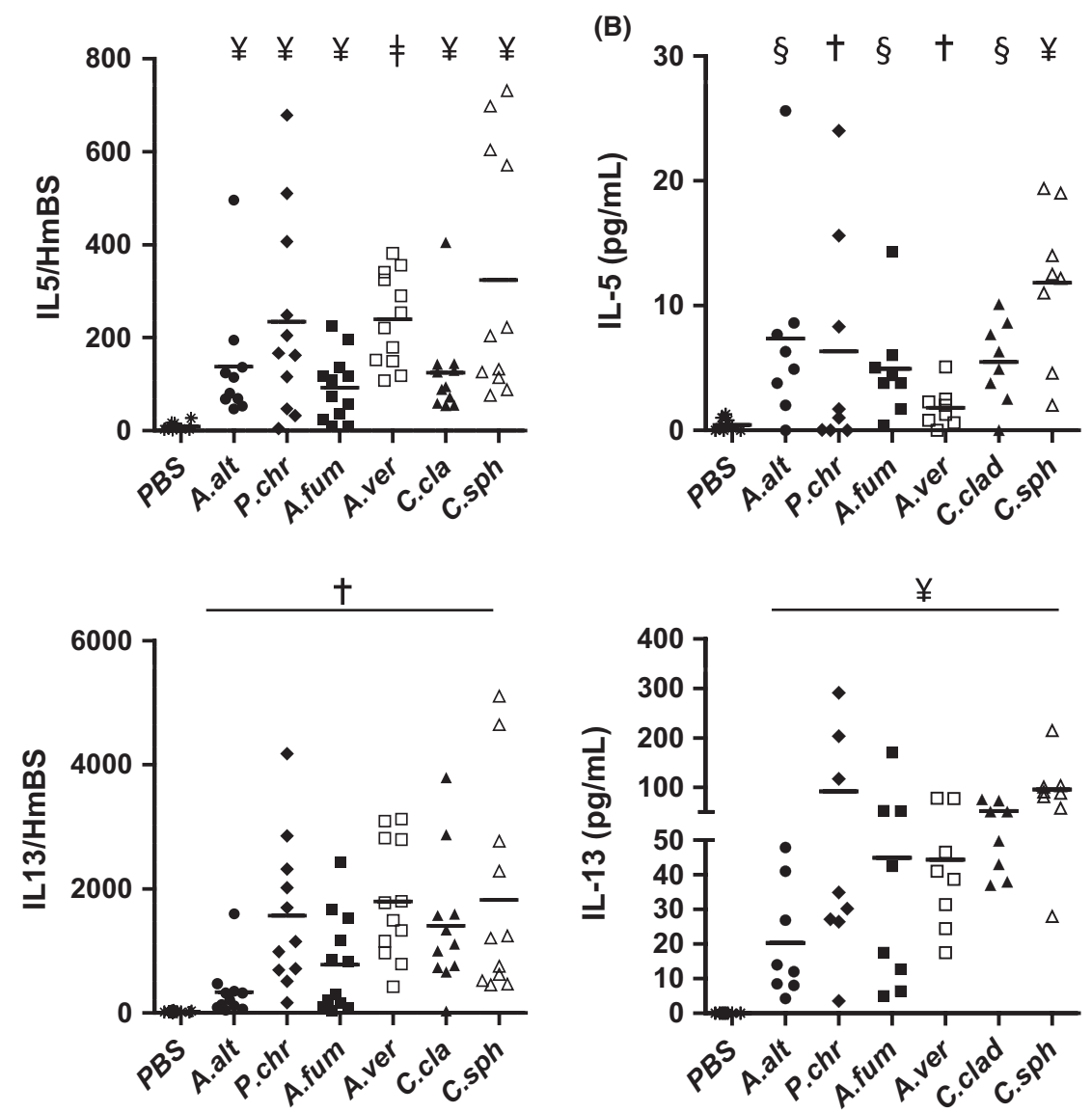

FIGURE 7 Analysis of Th2-type cytokine production after a chronic stimulation with mold spores. C57BL/6 mice were instilled daily during 3 days and then twice a week during 4 more weeks with PBS or various mold spores $\left(10^{6}\right.$ spores except $10^{5}$ spores for A. alternata). Mice were instilled again the next Monday. (A) The graphs show the lung transcriptional levels of the indicated Th2 cytokines and of IFN- $\gamma$ analyzed by qRT-PCR $24 \mathrm{~h}$ after the last instillation. Results are from two independent experiments with five to six individual mice per group. (B) IL-5 and IL13 concentrations in the BAL fluids $24 \mathrm{~h}$ after the last instillation. Results are from two independent experiments with five or six individual mice (PBS, A. fumigatus, A. versicolor, and C. cladosporioides group). Significant differences between mold-treated mice vs control PBS-treated mice $(† P<.05, \S P<.01, ¥ P<.001, \neq P<.0001)$

found that A. fumigatus was the most common "major sensitizer" (32\% of the positive sera) followed by $A$. alternata in $28 \%$ of the asthmatics. A. versicolor was not identified as the "major sensitizer" in any of the asthmatic patients. Other mold species were less frequently identified as "major sensitizer." Although when taken together, P. chrysogenum, P. brevicompactum, C. sphaerospermum, and C. cladosporioides were the "major sensitizers" for almost $30 \%$ of the asthmatics.

\section{4 | DISCUSSION}

The association between building dampness and respiratory pathologies is established. ${ }^{21,38,39}$ Additionally, recent studies are now pointing to a link between the presence of indoor molds and the development of asthma or the exacerbation of its symptoms. ${ }^{23,24,40}$ Here, we have analyzed and compared the inflammatory and allergenic properties 
FIGURE 8 Histopathological changes in lungs of mice chronically exposed to mold spores. C57BL/6 mice were instilled daily during 3 days and then twice a week during four more weeks with PBS or various mold spores $\left(10^{6}\right.$ spores except $10^{5}$ spores for A. alternata). Mice were instilled again the next Monday and $24 \mathrm{~h}$ later, lungs were collected, fixed in formalin, and prepared for histological examination. Left and central panels show representative lungs sections after hematoxylin-eosin-safran staining. Right panel shows representative lung sections after periodic acid Schiff staining. Mold species are indicated on the left side of the figure

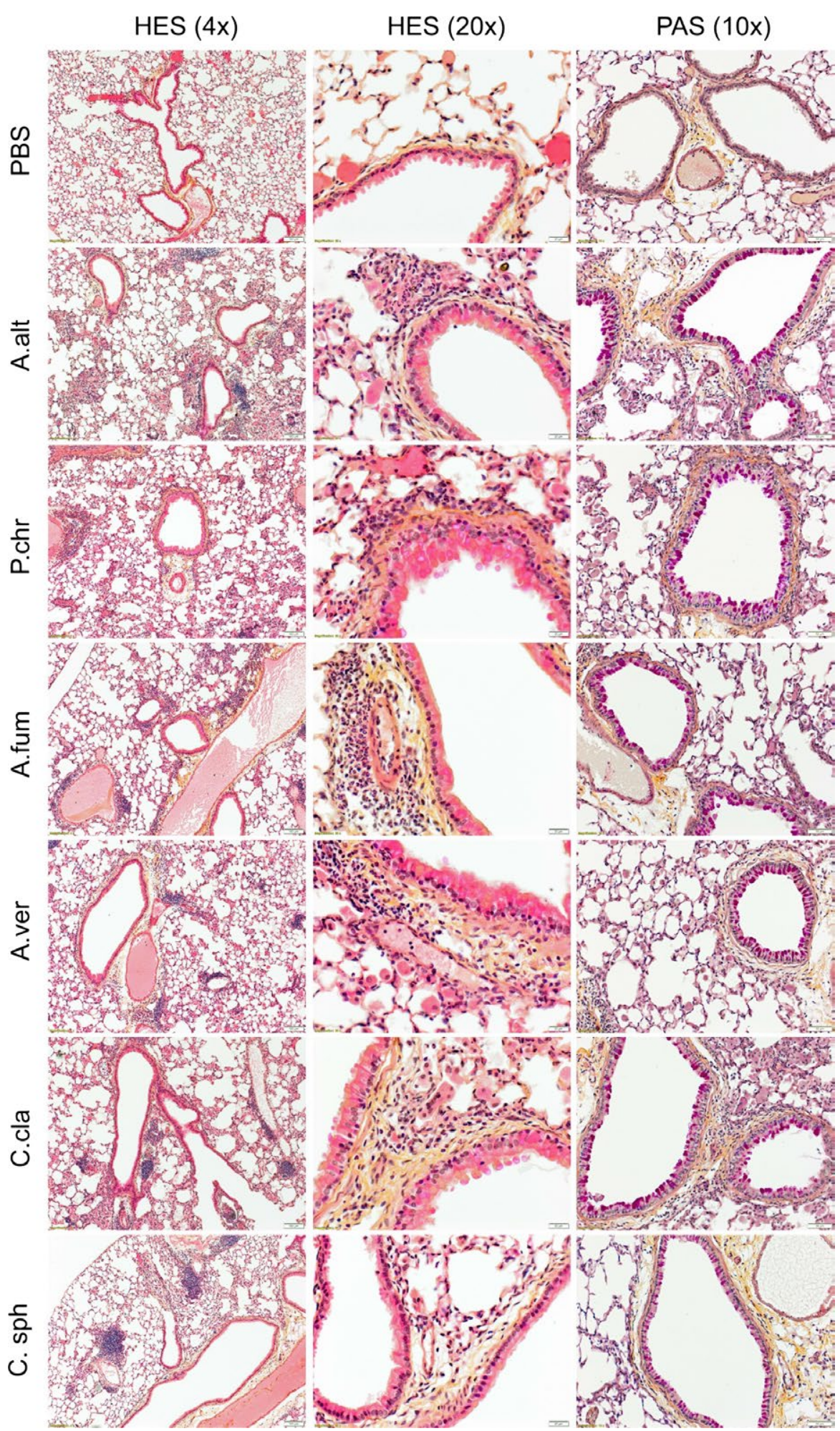

of six common mold species from indoor and outdoor environments. A. alternata is a typical outdoor mold while A. fumigatus is very common outdoors and indoors. P. chrysogenum, A. versicolor, C. cladosporïoides, and C. sphaerospermum are frequently found indoors, especially in damp dwellings. ${ }^{18,25,27,28,41}$

In vitro, spores from these six species elicited the secretion of inflammatory cytokines (TNF- $\alpha$, IL- 6, IL- $1 \alpha$ and IL-1 $\beta$ ) when cultured in the presence of murine BMDCs. The importance of $\mathrm{C}$-type lectins in the recognition of $\beta$-glucans exposed in germinating spores has been clearly established. ${ }^{42}$ In line with this, we found that this cytokine production was completely inhibited in MALT-1 (a caspase-like protease) deficient BMDCs. Indeed, cells that do not express MALT-1 are unable to form the CARD9-Bcl10-MALT1 complex downstream of the Dectin-Mincle receptors which subsequently hampers the activation of the NF-kB pathway (data not shown). 


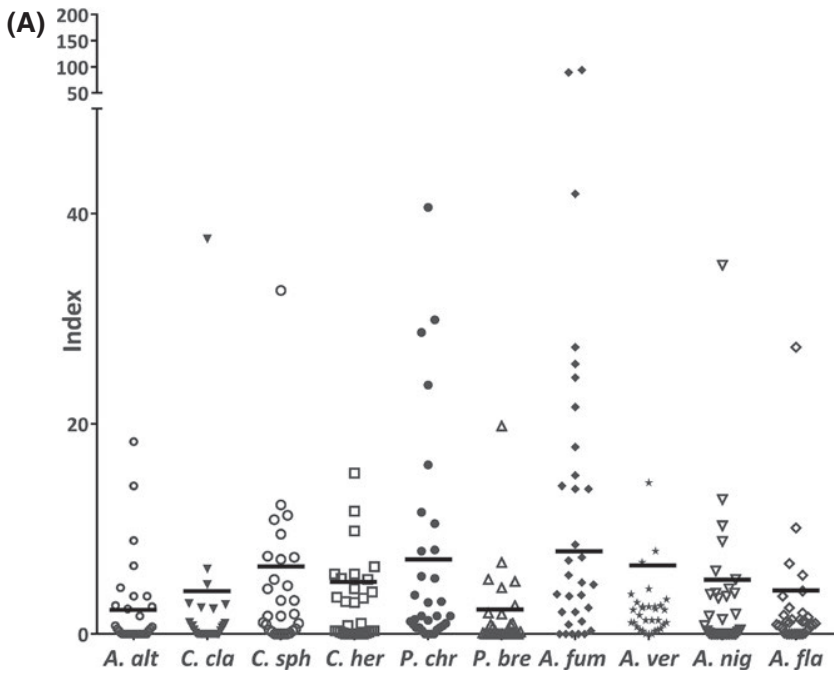

(B)

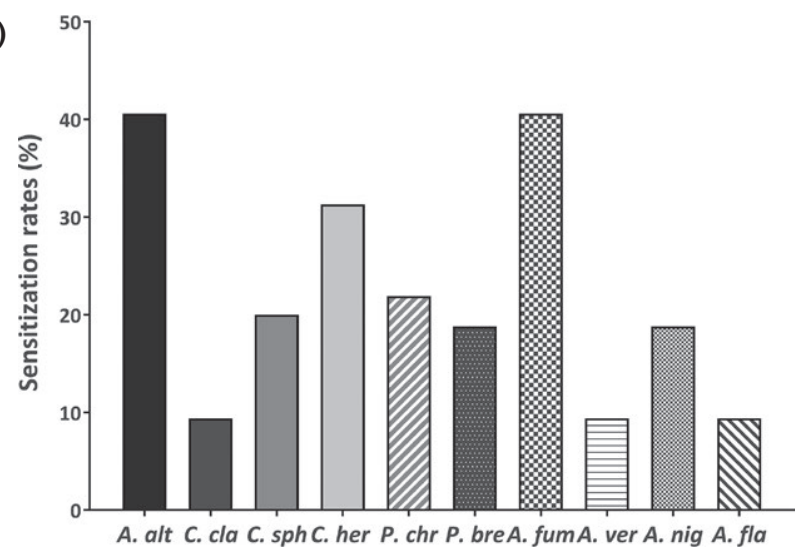

(C)

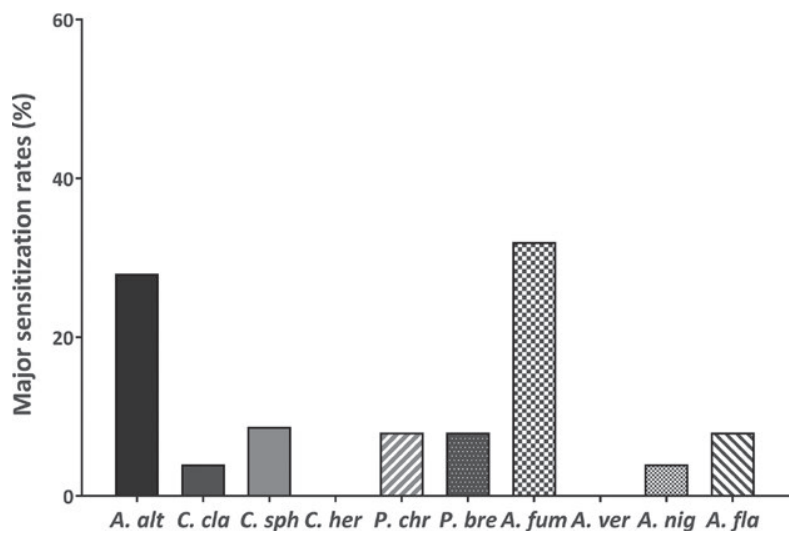

FIGURE 9 Sensitization frequencies against a panel of indoor and outdoor molds in mold positive SPT/CAP asthmatic patients. (A) The presence of specific lgE against 10 different mold species was determined in a total of 32 sera from asthmatic patients with a positive SPT/CAP for molds. Horizontal bars represent, for each mold, the positivity threshold. This threshold, calculated for each mold extract, corresponds to the mean response +3 standard deviations of a negative serum. (B) Sensitization frequencies (\%) for each mold of the panel ( $n=32)$. (C) Major sensitization frequencies (\%) for each mold of the panel. For each serum, this major sensitization was defined as the response against a single extract showing the highest difference as compared to the variation of response of the negative control serum $(n=25)$
In vitro, TNF- $\alpha$ production was induced by the different spores to comparable levels except for $A$. versicolor that only induced a low TNF- $\alpha$ secretion. However, when instilled into the lungs, three species, A. alternaria, A. versicolor, and C. sphaerospermum, induced a stronger early upregulation of lung TNF- $\alpha$ expression than the three other species. The TNF- $\alpha$ produced in vivo by inflammatory dendritic cells has previously been shown to shape the immune response during fungal infection and to promote neutrophilic airway inflammation. ${ }^{43}$ In our study, these three mold species also strongly upregulated the expression of lung CXCL-2 and induced an abundant early recruitment of neutrophils in the BAL fluids. Remarkably, for the six tested species, the recruitment of neutrophils in the BAL fluids was strongly correlated with the lung expression of CXCL-2. A. fumigatus also induced an early accumulation of numerous neutrophils into the lungs but with lower levels of lung TNF- $\alpha$ and CXCL-2. However, in vitro, A. fumigatus (and A. alternata) elicited a stronger secretion of IL- $1 \alpha$ and IL- $1 \beta$ than the other species. In several models, IL-1 has been shown to be able to stimulate the production of various neutrophil chemo-attractants. ${ }^{44,45}$ This axis might lie at the basis of the early neutrophil recruitment observed with $A$. fumigatus while A. alternata could trigger both TNF- $\alpha$ and IL-1 pathways. In contrast, $P$. chrysogenum spores only weakly recruited neutrophils in the BAL fluids and feebly upregulated the lung expression of inflammatory mediators indicating the weak inflammatory potential of this species.

In a previous report, we showed that spores from A. alternaria and C. herbarum injected in mice increased total IgE levels. ${ }^{31,32}$ Here, we found that five of the six mold species tested induced a rise of the IgE serum concentrations. Only A. versicolor failed to induce any IgE increase. This lack of IgE increase was also observed by Mintz-Cole et al. ${ }^{46}$ in their chronic model. An increase in serum IgE is usually indicative of a strong Th2 activation, secretion of IL-4 and/or IL-13 and STAT6 activation. ${ }^{47}$

We observed that spores of the six tested mold species led to an early upregulation of IL-33 expression when instilled in the lungs. This cytokine belongs to the IL-1 family and is rapidly upregulated in the lungs by some allergens e.a. A. alternaria. ${ }^{48,49}$ Its biological activity is increased in an inflammatory environment, due to the release of proteases cleaving the full length IL-33 into its active form. ${ }^{50} \mathrm{IL}-33$ initiates type- 2 immune response through its binding to the receptor T1/ST2 present on innate lymphoid cells, basophils, mast cells, eosinophils, and subsets of Th2 cells. ${ }^{50}$ Activated innate lymphoid cells vigorously produce IL-5 and IL-13 thereby creating an environment supporting the development of a Th2 response. ${ }^{48}$

Accordingly, a chronic treatment with spores from these six species led to the production of Th2 cytokines (IL4, IL-5, and IL-13) and a lung inflammation characterized by an accumulation of eosinophils in the BAL fluids. P. chrysogenum induced a strong recruitment of eosinophils with low neutrophil and macrophage numbers. For A. fumigatus, A.versicolor, and C.cladosporïoides, eosinophils outweighed neutrophils in the BAL fluids while A. alternaria and C. sphaerospermum spores recruited more neutrophils than eosinophils. Histological analysis of the lungs confirmed that $P$. chrysogenum, A. fumigatus, $A$. versicolor, and $C$. cladosporioides induced a recruitment of eosinophils in the 
(A)

FIGURE 10 Classification of mold species according to their immunomodulatory properties. (A) Classification established based on results obtained from mouse model experiments; (B) Classification established based on results obtained from investigations on mold sensitization rates in a group of moldsensitized asthmatics

(B)
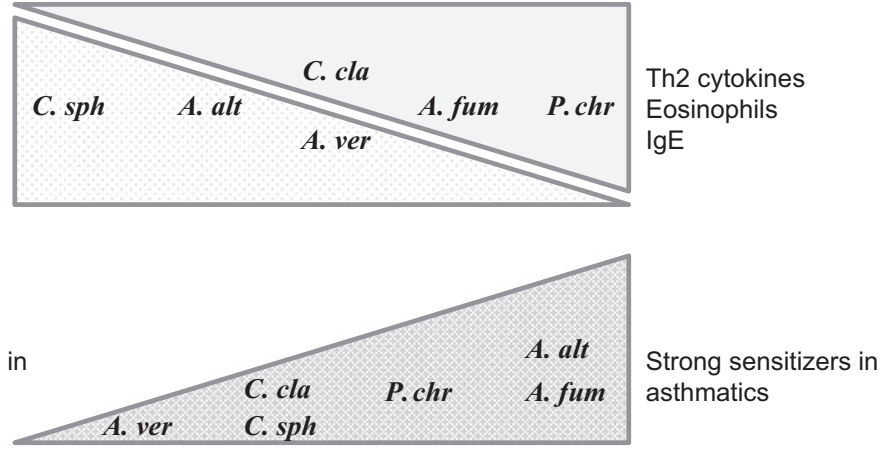

peri-bronchial areas and increased the mucus production (to a lesser extent for $C$. shaerospermum).

The ratio of IL-4/IFN- $\gamma$ production in the lung also confirmed the strong pro-Th2 potential of $P$. chrysogenum. However, this ratio is only partially representative of the Th2 response and other cytokines (IL-5 and IL-13) and chemokines (CCL-11 and CCL-24) should also be considered together with IL-17.

In the studies of Mintz-Cole et al., C. cladosporioides was described as a poor stimulator of the Th17 response due to a lack of beta-glucans present on the spore surface, whereas $A$. versicolor spores were shown to trigger dectin-1-dependent Th17 response leading to the development of a lung neutrophilic inflammation. ${ }^{49,51}$ We also detected the production of IL-17 in the lungs and BAL fluids of A. versicolor stimulated mice but in our model this response was associated with a production of Th2 cytokines and a lung eosinophilia. A difference between mold strains could not explain this inconsistency as two other A. versicolor strains were tested with similar results (data not shown). However, mold cultures are not easily standardized and differences in the media or in the preparation of the spores or differences in the protocols might account for these discrepancies. Moreover, we found that $C$. cladosporioides but also $C$. sphaerospermum were fully capable of stimulating lung production of IL-17, and thus, these three species induced mixed Th2/Th17 response when instilled chronically in the lungs of C57BL/6 mice. Th17 cells and their production of IL-23 are an integral part of the protective immunity against fungi, increasing antifungal responses by alveolar macrophages and neutrophils. ${ }^{52}$ IL17 can also upregulate the expression of $\mathrm{CXCL}$ chemokines leading to the recruitment of neutrophils, increase airway hyper-responsiveness and lies at the basis of the corticoid resistance of bronchial epithelial cells. ${ }^{53-55}$ An imbalance between Th2 and Th17 activation could therefore turn protective responses to pathology/allergy.

In addition to the responses triggered by the six tested mold species in mice, we also evaluated the production of specific IgE in a population of asthmatic patients sensitized to molds. We used a panel of 10 molds species that are known to be frequently isolated in Western Europe. ${ }^{25,28}$ In this population, we found IgE antibodies directed against all these mold species. In the context of mold sensitization, the cross-reactivity between various fungal allergens has been quite extensively reported. It generally involves fungi that are phylogenetically related and share homology in the amino acid sequence of epitopes but cross-reactivity can also involve distant species and carbohydrate determinants. ${ }^{56}$ Therefore, we calculated the "major reactivity" for each patient and found that $60 \%$ reacted against $A$. fumigatus and $A$. alternata. Other patients (28.7\%) had their major reactivity directed against the indoor species $P$. chrysogenum, $P$. brevicompactum, C. sphaerospermum, and C. cladosporïoides. These observations suggest that a genuine sensitization to indoor species can also play a role in the development of allergic asthma. Noteworthy, A. versicolor, albeit frequently isolated in dwellings, was never identified as "major sensitizer" in the group of asthmatics. Moreover, in mice, it failed to induce a significant IgE production.

Based on the results gathered in the murine model and in asthmatic patients, we aimed at establishing a ranking between rather inflammatory or rather allergenic mold species (Figure 10). Based on the mouse model results (early and late cytokine secretion profiles, cell recruitment in the BAL fluids and serum IgE production), species, such as $C$. sphaerospermum or A. alternata, seem to trigger inflammation rather than allergy. On the contrary, P. chrysogenum and A. fumigatus seem more pro-allergenic. The mixed profile of $A$. versicolor and $C$. cladosporioides was already addressed above. Of note, this classification should be considered as a continuum rather than a black or white distinction. In asthmatics, the profile is a bit different. As one could have expected from the mouse model results, $A$. fumigatus and $P$. chrysogenum emerged as strong sensitizers but A. alternata did so as well. This discrepancy for A. alternata between results obtained in the mouse model, and the sensitization of asthmatics has at least two potential explanations. Firstly, spores of A. alternata are much bigger (between 20 and $100 \mu \mathrm{mol} / \mathrm{L}$ ) than spores of other species (smaller than $10 \mu \mathrm{mol} / \mathrm{L}$ ) ${ }^{57}$ and they may possibly interact differently with the lung tissue in mice and humans. Secondly, because of their pro-cachectic effect, the concentration of spores of $A$. alternata used for lung instillation was ten times lower than for the other species. It is therefore possible that the allergenic properties of A. alternata were "attenuated" in our mouse model.

Our study has several limitations. Nasal instillations of mold spores (wet aerosol) do not represent the natural lung exposure to allergens in humans. Indeed, during physiological exposure, allergens settle in limited areas of the lungs where they can trigger an inflammatory response. ${ }^{18,58}$ After nasal instillation, such as in experimental models, allergens are widely spread in the bronchial tree and different mechanisms can be triggered explaining the apparent discrepancies. Another limitation of the study is the fact that the concentration of spores used in the mouse model that is needed to counter the lower natural 
susceptibility $\left(10^{5}-10^{6} /\right.$ instillation) is much higher than spore count usually present in the air (up to 20000 spores $/ \mathrm{m}^{3}$ for Cladosporium spp, up to 7500 spores $/ \mathrm{m}^{3}$ for A. alternata and much less for the other genus $^{1}$ ) and thus largely exceed natural concentration. Responses from the mouse model may therefore differ from responses observed in the asthmatic group where individuals have an increased sensitivity to mold exposure.

In summary, results from asthmatic patients show that species with an observed low allergenic potential in mice can be linked to asthma and require attention. Results from human and murine experiments have also shown that in addition to spores of the classical outdoor species A. alternata and A. fumigatus, spores from indoor mold species can also exert strong inflammatory and allergenic properties. Therefore, considering their harmful nature, future studies should focus on investigating their role in allergic diseases.

\section{ACKNOWLEDGEMENTS}

We thank the members of the laboratory of Pr. Yves Poumay (URPhym, NARILIS, Université de Namur) and especially Daniel Van Vlaender for his technical expertise for the lung preparation for histology analysis. This study was supported by the Belgian Science Policy (BELSPO) contract IAP P7/32 DISCOBEL "Molecular Signaling in Cell Death and Inflammation: an integrative approach from basic mechanisms to disease models" and by an Ylieff fellowship.

\section{REFERENCES}

1. Twaroch TE, Curin M, Valenta R, Swoboda I. Mold allergens in respiratory allergy: from structure to therapy. Allergy Asthma Immunol Res. 2015;7:205-220.

2. Drummond RA, Saijo S, Iwakura Y, Brown GD. The role of Syk/ CARD9 coupled C-type lectins in antifungal immunity. Eur J Immunol. 2011;41:276-281.

3. Werner JL, Metz AE, Horn D, et al. Requisite role for the dectin-1 beta-glucan receptor in pulmonary defense against Aspergillus fumigatus. J Immunol. 2009;182:4938-4946.

4. Rivera A. Protective immune responses to fungal infections. Parasite Immunol. 2014;36:453-462.

5. Rivera A, Hohl TM, Collins N, et al. Dectin-1 diversifies Aspergillus fumigatus-specific $\mathrm{T}$ cell responses by inhibiting T helper type $1 \mathrm{CD} 4$ T cell differentiation. J Exp Med. 2011;208:369-381.

6. Romani L. Immunity to fungal infections. Nat Rev Immunol. 2011;11:275-288

7. Free SJ. Fungal cell wall organization and biosynthesis. Adv Genet. 2013;81:33-82.

8. Horner WE, Helbling A, Salvaggio JE, Lehrer SB. Fungal allergens. Clin Microbiol Rev. 1995;8:161-179.

9. Vijay HM, Kurup VP. Fungal allergens. Clin Allergy Immunol. 2008;21:141-160.

10. Scheurer S, Toda M, Vieths S. What makes an allergen? Clin Exp Allergy. 2015;45:1150-1161.

11. Shakib F, Ghaemmaghami AM, Sewell HF. The molecular basis of allergenicity. Trends Immunol. 2008;29:633-642.

12. Umetsu DT, McIntire JJ, Akbari O, Macaubas C, DeKruyff RH. Asthma: an epidemic of dysregulated immunity. Nat Immunol. 2002;3:715-720.

13. Barnes PJ. Pathophysiology of allergic inflammation. Immunol Rev. 2011;242:31-50.
14. Lambrecht $\mathrm{BN}$, Hammad $\mathrm{H}$. The immunology of asthma. Nat Immunol. 2015;16:45-56.

15. Al-Ramli W, Prefontaine D, Chouiali F, et al. T(H)17-associated cytokines (IL-17A and IL-17F) in severe asthma. J Allergy Clin Immunol. 2009;123:1185-1187.

16. Zureik M, Neukirch C, Leynaert B, Liard R, Bousquet J, Neukirch F. Sensitisation to airborne moulds and severity of asthma: cross sectional study from European Community respiratory health survey. BMJ. 2002;325:411-414.

17. Black PN, Udy AA, Brodie SM. Sensitivity to fungal allergens is a risk factor for life-threatening asthma. Allergy. 2000;55:501-504.

18. Nevalainen A, Taubel M, Hyvarinen A. Indoor fungi: companions and contaminants. Indoor Air. 2015;25:125-156.

19. Portnoy JM, Barnes CS, Kennedy K. Importance of mold allergy in asthma. Curr Allergy Asthma Rep. 2008;8:71-78.

20. Choi H, Byrne S, Larsen LS, et al. Residential culturable fungi, (1-3, 1-6)-beta-d-glucan, and ergosterol concentrations in dust are not associated with asthma, rhinitis, or eczema diagnoses in children. Indoor Air. 2014;24:158-170.

21. Fisk WJ, Eliseeva EA, Mendell MJ. Association of residential dampness and mold with respiratory tract infections and bronchitis: a metaanalysis. Environ Health. 2010;9:72.

22. Mendell MJ, Mirer AG, Cheung K, Tong M, Douwes J. Respiratory and allergic health effects of dampness, mold, and dampness-related agents: a review of the epidemiologic evidence. Environ Health Perspect. 2011;119:748-756.

23. Behbod B, Sordillo JE, Hoffman EB, et al. Asthma and allergy development: contrasting influences of yeasts and other fungal exposures. Clin Exp Allergy. 2015;45:154-163.

24. Sharpe RA, Bearman N, Thornton CR, Husk K, Osborne NJ. Indoor fungal diversity and asthma: a meta-analysis and systematic review of risk factors. J Allergy Clin Immunol. 2015;135:110-122.

25. Beguin $\mathrm{H}$. Mould biodiversity in homes. I. Air and surfaces analysis of 130 dwellings. Aerobiologia. 1994;10:157-166.

26. Bellanger AP, Reboux G, Roussel S, et al. Indoor fungal contamination of moisture-damaged and allergic patient housing analysed using realtime PCR. Lett Appl Microbiol. 2009;49:260-266.

27. Horner WE, Worthan AG, Morey PR. Air- and dustborne mycoflora in houses free of water damage and fungal growth. Appl Environ Microbiol. 2004;70:6394-6400.

28. Reboux G, Bellanger AP, Roussel S, et al. Indoor mold concentration in Eastern France. Indoor Air. 2009;19:446-453.

29. Achatz G, Oberkofler H, Lechenauer E, et al. Molecular cloning of major and minor allergens of Alternaria alternata and Cladosporium herbarum. Mol Immunol. 1995;32:213-227.

30. Inaba K, Inaba M, Romani N, et al. Generation of large numbers of dendritic cells from mouse bone marrow cultures supplemented with granulocyte/macrophage colony-stimulating factor. J Exp Med. 1992;176:1693-1702.

31. Denis O, van den Brule S, Heymans J, et al. Chronic intranasal administration of mould spores or extracts to unsensitized mice leads to lung allergic inflammation, hyper-reactivity and remodelling. Immunology. 2007;122:268-278.

32. Havaux X, Zeine A, Dits A, Denis O. A new mouse model of lung allergy induced by the spores of Alternaria alternata and Cladosporium herbarum molds. Clin Exp Immunol. 2005;139:179-188.

33. Korf JE, Pynaert G, Tournoy K, et al. Macrophage reprogramming by mycolic acid promotes a tolerogenic response in experimental asthma. Am J Respir Crit Care Med. 2006;174:152-160.

34. GINA: Global Initiative for Asthma: Global Strategy for Asthma Management and Prevention. In 2014. 2014.

35. Vincent M, Romano M, Corazza F, Huygen K, Michel O, Denis O. Development of a dot-blot assay for the detection of mould-specific IgE in the Belgian population. Mycopathologia. 2016;182:319-329. 
36. Crameri R, Garbani M, Rhyner C, Huitema C. Fungi: the neglected allergenic sources. Allergy. 2014;69:176-185.

37. Soeria-Atmadja D, Onell A, Borga A. IgE sensitization to fungi mirrors fungal phylogenetic systematics. J Allergy Clin Immunol. 2010;125:1379-1386

38. Jaakkola MS, Quansah R, Hugg TT, Heikkinen SA, Jaakkola JJ. Association of indoor dampness and molds with rhinitis risk: a systematic review and meta-analysis. J Allergy Clin Immunol. 2013;132:1099-1110.

39. Quansah R, Jaakkola MS, Hugg TT, Heikkinen SA, Jaakkola JJ. Residential dampness and molds and the risk of developing asthma: a systematic review and meta-analysis. PLoS One. 2012;7:e47526.

40. Karvonen AM, Hyvarinen A, Korppi M, et al. Moisture damage and asthma: a birth cohort study. Pediatrics. 2015;135:e598-e606.

41. Rocchi S, Reboux G, Frossard V, et al. Microbiological characterization of 3193 French dwellings of Elfe cohort children. Sci Total Environ. 2015;505:1026-1035.

42. Hardison SE, Brown GD. C-type lectin receptors orchestrate antifungal immunity. Nat Immunol. 2012;13:817-822.

43. Fei M, Bhatia S, Oriss TB, et al. TNF-alpha from inflammatory dendritic cells (DCs) regulates lung IL-17A/IL-5 levels and neutrophilia versus eosinophilia during persistent fungal infection. Proc Natl Acad Sci USA. 2011;108:5360-5365.

44. Lee YS, Yang H, Yang JY, et al. Interleukin-1 (IL-1) signaling in intestinal stromal cells controls $\mathrm{KC} / \mathrm{CXCL} 1$ secretion, which correlates with recruitment of IL-22- secreting neutrophils at early stages of Citrobacter rodentium infection. Infect Immun. 2015;83:3257-3267.

45. van de Veerdonk FL, Joosten LA, Netea MG. The interplay between inflammasome activation and antifungal host defense. Immunol Rev. 2015;265:172-180.

46. Mintz-Cole RA, Gibson AM, Bass SA, Budelsky AL, Reponen T, Hershey GK. Dectin-1 and IL-17A suppress murine asthma induced by Aspergillus versicolor but not Cladosporium cladosporioides due to differences in beta-glucan surface exposure. J Immunol. 2012;189:3609-3617.

47. Shimoda K, van Deursen J, Sangster MY, et al. Lack of IL-4-induced Th2 response and IgE class switching in mice with disrupted Stat6 gene. Nature. 1996;380:630-633.

48. Bartemes KR, lijima K, Kobayashi T, Kephart GM, McKenzie AN, Kita H. IL-33-responsive lineage- CD25+ CD44(hi) lymphoid cells mediate innate type 2 immunity and allergic inflammation in the lungs. $J$ Immunol. 2012;188:1503-1513.

49. Denis O, Vincent M, Havaux X, De PS, Treutens G, Huygen K. Induction of the specific allergic immune response is independent of proteases from the fungus Alternaria alternata. Eur J Immunol. 2013:43:907-917.

50. Lefrancais E, Roga S, Gautier V, et al. IL-33 is processed into mature bioactive forms by neutrophil elastase and cathepsin G. Proc Natl Acad Sci USA. 2012;109:1673-1678.

51. Mintz-Cole RA, Brandt EB, Bass SA, Gibson AM, Reponen T, Khurana Hershey GK. Surface availability of beta-glucans is critical determinant of host immune response to Cladosporium cladosporioides. J Allergy Clin Immunol. 2013;132:159-169.

52. Chamilos G, Ganguly D, Lande R, et al. Generation of IL-23 producing dendritic cells (DCs) by airborne fungi regulates fungal pathogenicity via the induction of T(H)-17 responses. PLoS One. 2010;5:e12955.

53. McKinley L, Alcorn JF, Peterson A, et al. TH17 cells mediate steroidresistant airway inflammation and airway hyperresponsiveness in mice. J Immunol. 2008;181:4089-4097.

54. Newcomb DC, Peebles RS Jr. Th17-mediated inflammation in asthma. Curr Opin Immunol. 2013;25:755-760.

55. Zijlstra GJ, Ten Hacken NH, Hoffmann RF, van Oosterhout AJ, Heijink IH. Interleukin-17A induces glucocorticoid insensitivity in human bronchial epithelial cells. Eur Respir J. 2012;39:439-445.

56. Crameri R, Zeller S, Glaser AG, Vilhelmsson M, Rhyner C. Crossreactivity among fungal allergens: a clinically relevant phenomenon? Mycoses. 2009;52:99-106.

57. Simon-Nobbe B, Denk U, Poll V, Rid R, Breitenbach M. The spectrum of fungal allergy. Int Arch Allergy Immunol. 2008;145:58-86.

58. Platts-Mills TA, Schuyler AJ, Erwin EA, Commins SP, Woodfolk JA. IgE in the diagnosis and treatment of allergic disease. J Allergy Clin Immunol. 2016;137:1662-1670.

\section{SUPPORTING INFORMATION}

Additional Supporting Information may be found online in the supporting information tab for this article.

How to cite this article: Vincent M, Percier P, De Prins S, et al. Investigation of inflammatory and allergic responses to common mold species: Results from in vitro experiments, from a mouse model of asthma, and from a group of asthmatic patients. Indoor Air. 2017;27:933-945. https://doi.org/10.1111/ina.12385 